DOI: http://dx.doi.org/10.12957/demetra.2014.8707

\title{
Adesão às recomendações alimentares de usuários portadores de diabetes tipo 2
}

\section{Compliance with dietary recommendations for users with type 2 diabetes}

Camila lrigonhé Ramos'

Jocilei Carniato Klug ${ }^{2}$

Alessandra Doumid Borges Pretto ${ }^{3}$

1 Programa de Pós-graduação em Nutrição e Alimentos. Universidade Federal de Pelotas. Pelotas, RS, Brasil.

${ }^{2}$ Faculdade de Nutrição. Universidade Federal de Pelotas. Pelotas, RS, Brasil.

3 Programa de Pós-graduação, Saúde e Comportamento. Universidade Católica de Pelotas. Pelotas, RS, Brasil.

Correspondência / Correspondence

Camila Irigonhé Ramos

E-mail:mila85@gmail.com

\section{Resumo}

Introdução: o diabetes mellitus (DM) é uma doença crônica não transmissível e impõe ao indivíduo mudanças importantes no seu cotidiano. Para manter a doença controlada, faz-se necessário que os portadores alterem e integrem a suas atividades diárias hábitos de alimentação saudáveis, muitas vezes diferindo do consumo diário. A adesão ao plano alimentar torna-se uma questão-chave para a qualidade de vida e o sucesso do tratamento. Objetivo: entender a adesão às recomendações alimentares de portadores de DM tipo 2 cadastrados no Programa Hiperdia de uma Unidade Básica de Saúde, em Pelotas-RS. Metodologia: a coleta de dados ocorreu por meio de entrevistas semiestruturadas, realizadas com 16 pessoas. Os dados foram analisados e interpretados utilizandose o processo de categorização de unidades de significado, identificando-se os temas significativos e reveladores da adesão às recomendações alimentares. Resultados: a não adesão às orientações alimentares ocorre, principalmente, por dificuldades em recriar hábitos alimentares, devido à não aceitação das restrições alimentares recebidas. Conclusão: as restrições alimentares acarretam mudanças negativas no convívio social e lacunas sobre o conhecimento da doença, com a consequente necessidade de um novo comportamento alimentar. Destacam a necessidade de trabalhar no serviço de saúde, com profundidade, as orientações e a adesão dos usuários.

Palavras-chave: Adesão. Recomendações Alimentares. Diabetes Mellitus. Hábitos Alimentares. 


\section{Abstract}

Introduction: diabetes mellitus (DM) is a chronic non-communicable disease and imposes on individuals major changes in their daily lives. To keep the disease under control, it is necessary that carriers change and integrate their daily activities habits of healthy eating, often differing from daily consumption. The adherence to diet becomes a key issue for quality of life and successful treatment. Objective: To understand compliance with dietary recommendations in patients with type 2 diabetes enrolled in the program Hiperdia at a Basic Health Unit in the city of Pelotas-RS. Methodology: Data was collected through semi-structured interviews with 16 people, analyzed and interpreted using the process of categorizing units of meaning, identifying the significant issues and reveal the compliance with dietary recommendations. Results: non-compliance with dietary guidelines occurs mainly by difficulties in recreating eating habits due to non-acceptance of received dietary restrictions. Conclusion: dietary restrictions have negative changes in social interaction and knowledge gaps about the disease, with the consequent need for a new food behavior. They highlight the need to deeply work guidelines and membership of users in health care services.

Key words: Compliance. Dietary Recommendations. Diabetes Mellitus. Food Habits.

\section{Introdução}

O diabetes mellitus (DM) é um dos mais importantes problemas de saúde pública e se destaca tanto pelo número de pessoas afetadas, quanto pela mortalidade prematura. ${ }^{1,2}$ Estima-se que, de acordo com as taxas atuais de morbimortalidade e as taxas de prevalência de DM tipo 2, haverá um aumento crescente desta doença, em todas as faixas etárias em países de baixa e média renda. ${ }^{3}$ Para o continente americano, projeta-se que cerca de 64 milhões de pessoas desenvolvam DM até 2025. ${ }^{3}$ Nos países de alta renda, esse aumento se evidencia nas faixas etárias mais avançadas, em consequência do aumento da expectativa de vida e do crescimento populacional. Nos países de baixa e média renda, esse aumento deverá ocorrer em todas as idades, principalmente no grupo de 45-64 anos, cuja prevalência será triplicada. ${ }^{3}$ No Brasil, o DM já apresenta uma prevalência de $8 \%$ nesta última faixa etária. ${ }^{4}$ 
O sistema público de saúde, no contexto nacional, enfrenta desafios importantes em relação ao DM, como por exemplo, garantir o acompanhamento terapêutico sistemático dos indivíduos portadores da doença; desenvolver ações visando à promoção da saúde e promover efetivamente a prevenção. Para firmar essas ações, em 2000, o Ministério da Saúde brasileiro implementou o Plano de Reorganização da Atenção à Hipertensão Arterial e ao Diabetes Mellitus. ${ }^{5} \mathrm{O}$ objetivo do plano é estabelecer diretrizes e metas para a reorganização no Sistema Único de Saúde (SUS), investindo na atualização dos profissionais da Atenção Básica, oferecendo garantia do diagnóstico ao usuário e proporcionando sua vinculação às Unidades de Saúde para tratamento, e acompanhamento continuado. ${ }^{5}$

O DM, por ser uma doença crônica não transmissível, impõe ao indivíduo mudanças importantes no seu cotidiano. Para manter a doença controlada, faz-se necessário que os doentes alterem e integrem, a suas atividades diárias, hábitos de alimentação saudáveis, muitas vezes diferindo do tipo de consumo diário. ${ }^{6}$ A adesão ao plano alimentar torna-se uma questão-chave para a qualidade de vida dos indivíduos com DM e o sucesso do tratamento. ${ }^{7}$ Contudo, estudos mostraram que modificar o estilo de vida é uma tarefa bastante complexa. ${ }^{6}$

A não adesão é um comportamento esperado em terapêuticas de doenças que exigem cuidados especiais e/ou mudanças de comportamento. Estudos mostram que indivíduos com DM, em geral, não são aderentes a todo o tratamento recomendado. ${ }^{8,9}$ Entre as explicações para esse fato, encontram-se a oferta de orientações com baixa efetividade, dificuldades de adaptação aos horários para as refeições, ${ }^{8}$ problemas para evitar os doces ${ }^{1,9}$ e modificar o preparo das refeições ${ }^{9}$ Na maioria das vezes, a não adesão ao tratamento do DM está vinculada a fatores socioeconômicos, psicossociais e culturais. ${ }^{1,6,7}$

Considerando a importância das recomendações alimentares para a saúde dos portadores de DM, este artigo teve a intenção de compreender as razões da não adesão de portadores de DM em tratamento, em uma Unidade Básica de Saúde (UBS) do sul do país, utilizando, para isso, a abordagem qualitativa de pesquisa.

\section{Métodos}

Tendo em vista o objetivo desta investigação, a opção metodológica foi pela abordagem qualitativa. $\mathrm{O}$ universo estudado foi composto por 16 entrevistados adultos (sete homens e nove mulheres), com média de idade de 45 anos entre adultos e de 65 anos entre idosos, sendo que a média não variou entre os sexos. Todos os sujeitos entrevistados possuíam diagnóstico médico de DM, estavam em tratamento em uma UBS do município de Pelotas-RS e eram cadastrados no Programa Hiperdia. 
A escolha da UBS deve-se à especificação da sua equipe, na qual se encontram professores e estagiários da Faculdade de Nutrição, além de médicos, enfermeiros, nutricionista, técnicos e ou auxiliares de enfermagem.

Após conhecimento e autorização da coordenação da UBS, os portadores de DM2 foram contatados e, tendo o consentimento escrito (todos os participantes assinaram o Termo de Conhecimento Livre e Esclarecido) e oral dos sujeitos para participar da pesquisa, iniciou-se a coleta dos dados face a face com os usuários e, em paralelo, as informações do cadastro do Hiperdia foram colhidas. As informações pessoais coletadas do cadastro foram: idade, sexo, endereço e dados relacionados à saúde (medicamentos utilizados, comorbidades e antecedentes familiares de doenças crônicas).

Pela inviabilidade de acessar todos os cadastrados, o universo de pesquisa foi composto por todos os adultos cujo grupo se reunia com periodicidade regular de uma vez ao mês. A escolha do dia/grupo deu-se por conveniência, fato que não impediu que a diversidade (idade, sexo, condições de saúde) estivesse presente também neste grupo.

Dos 50 indivíduos cadastrados, apenas 20 foram convidados a participar do estudo no primeiro encontro, visto que 30 deles não compareceram na data agendada para reunião do grupo, nem à reunião agendada subsequentemente. Portanto, o estudo contou com o depoimento de todos os participantes presentes em dois dias de reunião do Hiperdia e que aceitaram fazer parte da pesquisa. Destes 20, houve quatro perdas, sendo três por não encontrarmos o domicílio e um por óbito. Dessa forma, a amostra totalizou 16 usuários.

A maioria das entrevistas ocorreu nas residências dos participantes; apenas quatro foram feitas na UBS. Os informantes elegíveis obedeceram aos seguintes critérios de inclusão: ter $\geq 18$ anos de idade, ter diagnóstico médico de DM, estar em tratamento em uma UBS do município de Pelotas-RS, ser cadastrados no Programa Hiperdia e estar ou ter algum familiar presente em um dia de reunião, quando ocorrem a entrega dos medicamentos e as orientações alimentares. Esse critério foi feito para confirmar o acesso deste grupo a medicamentos e profissionais e as relações com um possível comportamento não aderente.

Os nomes dos participantes foram mantidos em sigilo, e codinomes foram utilizados na explanação dos resultados, respeitando-se a Resolução n 196/96 para pesquisas envolvendo seres humanos, do Conselho Nacional de Saúde, Ministério da Saúde. ${ }^{10} \mathrm{O}$ estudo foi aprovado pelo Comitê de Ética em Pesquisa da Faculdade de Medicina, da Universidade Federal de Pelotas UFPel (Protocolo n ${ }^{\circ}$ 025/08).

Foram realizadas duas entrevistas semiestruturadas com cada participante, com duração de $40 \mathrm{~min}$ a 1 h30min, com intervalo de um a dois meses, focando nas práticas que favorecem, ou 
não, a adesão às recomendações alimentares. As entrevistas realizaram-se entre dezembro de 2008 e março de 2009. O material foi gravado e transcrito para análises posteriores por meio da identificação de unidades de significado. A intenção foi compreender o fenômeno da adesão ou não, a partir da categorização de temas emergentes, e entender como as práticas favorecem ou não a adesão às recomendações alimentares e ao tratamento adequado para DM.

\section{Análise dos resultados}

Foram seguidos os critérios de confiabilidade, coerência e significado, segundo Wise. ${ }^{11} \mathrm{~A}$ confiabilidade foi alcançada pela explicitação dos passos dados na abordagem aos participantes, pela documentação de todo o material e pela validação dos achados pelos sujeitos de estudo. A coerência do texto foi garantida por meio das respostas às questões de pesquisa. O significado foi dado pela fidelidade às experiências vividas pelos sujeitos investigados, pois são reais e nascidas das vivências dos participantes.

\section{Resultados e discussão}

É de suma importância que os profissionais de saúde estejam preparados para compreender os desafios da interação com portadores do DM, visto que a relação médico-paciente afeta a adesão às recomendações alimentares. ${ }^{11}$ Traverso-Yépez, em estudo realizado com usuários da rede básica, em Natal-RN, demonstrou que os entrevistados buscavam atendimento de qualidade baseado na escuta, diálogo, atenção e respeito. ${ }^{12}$

As instituições públicas são referidas como aquelas cujo usuário é, muitas vezes, visto como um depositário ou gerador de problemas de saúde. ${ }^{13,14}$ Esse tipo de relação entre os profissionais e os usuários não propicia uma boa cumplicidade, necessária para o usuário sentir-se acolhido e à vontade para expor suas dúvidas e comportamentos. Segundo Gonçalves et al., "muitos pacientes, com doenças crônicas, despendem uma parte de suas vidas no 'papel de paciente' e, nestes casos, a relação médico-paciente é um dos fatores importantes na decisão de seguir ou não as recomendações médicas". ${ }^{15}$

Para melhor compreender alguns dos fatores que influenciaram na aderência às recomendações alimentares, os dados representativos dos temas que estruturam o fenômeno da adesão foram apresentados conforme as dúvidas em relação à etiologia e o desenvolvimento do DM, as informações e alguns fatores que interferiram na adesão às recomendações alimentares. 


\section{Causa e desenvolvimento do DM}

O DM desenvolve-se de maneira silenciosa, o que faz com que muitos portadores desta doença desconheçam estar doentes e, consequentemente, demorem a buscar o serviço de saúde. ${ }^{16} \mathrm{~A}$ demora na busca pelo tratamento, bem como a falta de comunicação entre o profissional de saúde e o usuário, podem desencadear a piora no quadro de saúde do portador de DM. Após a descoberta da doença, o entendimento das causas que a desencadearam é um dos fatores que contribuem para a aceitação da patologia e maior adesão ao tratamento. ${ }^{16}$ Welfer e Leite, em estudo no município de Ijuí-RS, ressaltaram a importância de fornecer informações ao usuário que lhe permitam entender a necessidade de alterar o estilo de vida para a promoção, manutenção e recuperação da saúde. ${ }^{17}$

Ao serem questionados sobre seu conhecimento a respeito do DM, a maioria dos entrevistados do estudo relatou ter apenas informações sobre as restrições de consumo alimentar impostas pela doença: "Eu sabia que minha prima tinha essa doença e que por causa disso não podia comer um monte de coisa!" (Hilda, 64 anos). "Um amigo meu teve que amputar a perna por causa do DM, dai já se vê como é séria essa doença” (Heitor, 46 anos).

No caso relatado, a descoberta do DM ocorreu quase que por acaso, muitas ao realizar tratamento para outras patologias. Entre os indivíduos contatados, observou-se que os sintomas e a causa da doença são desconhecidos, dificultando a prevenção e procura imediata do serviço. Além disso, o desconhecimento sobre as consequências da doença pode afetar com mais força a adesão às restrições da dieta habitual, visto que estas exigem modificações de hábitos alimentares e familiares bastante arraigados.

Em relação à causa do desenvolvimento do DM, alguns estudos com abordagem qualitativa encontraram dúvidas e comportamentos similares. Maias Neto demonstrou que ocorre ausência de conhecimento sobre a doença ao estudar experiências e percepções de sujeitos portadores de DM. ${ }^{18}$ Dados semelhantes, encontrados por Welfer, apontaram que, antes do diagnóstico, muitos portadores da doença desconheciam ser diabéticos e não distinguiam os sinais e sintomas da doença. Em razão disso, eles demoraram a procurar o serviço de saúde e iniciar seu tratamento. ${ }^{17}$

Uma participante deste estudo (Carolina, 59 anos), ao contar sobre os sintomas que apresentava no momento do diagnóstico, relatou que a visão estava embaçada, em estágio de quase cegueira quando foi à UBS procurar atendimento médico para tratamento de uma gripe. Ela conta que não entende por que desenvolveu o DM e refere achar que possa ter surgido de problemas emocionais devido à perda de seu esposo na época em que recebeu o diagnóstico. As explicações sobre esta relação não foram aprofundadas neste estudo. Todavia, existem muitos estudos que investigam as explicações causais do aparecimento da doença, mostrando que a lógica dos indivíduos doentes difere da biomédica, levando os tratamentos a adaptações nem sempre favoráveis à cura ou ao controle da doença. ${ }^{17,18}$ 
Outro informante (Heitor, 46 anos), quando questionado sobre os fatores que o levaram a desenvolver o DM, modificou o tom de voz ao responder. A conversa ficou tensa e ele enfatizou angustiado que ainda desconhece vários aspectos sobre essa doença. Heitor então questionou: "Por que essa doença apareceu?" e interpelou a entrevistadora, perguntando se ela iria lhe esclarecer o motivo como um desabafo e um pedido de explicação. Há sete anos, ele descobriu ter DM e começou o tratamento medicamentoso em seguida, mas há um ano parou com a medicação, por conta própria, por se sentir melhor e não apresentar sintomas do DM. Após esta conduta, sua glicemia chegou a $600 \mathrm{mg} \backslash \mathrm{dL}$ e ele entrou em coma diabético. Após esse episódio, Heitor começou com o uso da insulina juntamente com a medicação. Voltou à UBS para adequar sua alimentação e, desde o ocorrido, adere corretamente ao tratamento medicamentoso e às orientações alimentares.

Já Fernanda (63 anos) relata que está habituada ao tratamento da doença e segue as prescrições relativas à dieta, mas apesar de se tratar há mais de 15 anos com médicos, nunca escutou uma explicação referente à causa de seu DM. A partir do diagnóstico, alguns portadores de DM se questionaram sobre a origem da doença; alguns acreditam que possa ser hereditário ou emocional, mas não compreendem ao certo o que esses fatores significam, nem como eles influenciam no surgimento da doença. Todavia, a hereditariedade foi mais bem aceita, porque os portadores de DM identificaram parentes consanguíneos que tinham ou têm a doença. Essa situação é exposta por Maias Neto ${ }^{18}$ e pode ser observada nos relatos que se seguem:

Minha mãe era diabética e a minha irmã também. Até o médico me perguntou se tinha alguém na família com diabetes. Que [a DM] tem fator de família, pra ter diabetes, vai passando de pai pra filho, ele me explicou. (Carlos, 56 anos).

Me lembro dos médicos dizerem que era da família, porque na minha família tinha diabéticos. Por isso, constato que meu diabetes veio passando pela família. (Maria, 69 anos).

Em seus relatos, todos os portadores de DM revelaram que não há esclarecimento, por parte dos profissionais da saúde, sobre as causas e os sintomas do DM. Em muitos casos, esta prática dificulta a aceitação da doença e seu tratamento. Estudos apontam para a necessidade de reflexão sobre a humanização das relações na prática da medicina..$^{19,20}$

Caprara, analisando a relação entre médicos e pacientes da equipe de Saúde da Família, enfatizou que, na comunicação entre ambos, surgem problemas importantes, como o fato de os médicos não explicarem de forma clara e compreensiva a patologia ao doente; e ainda, por esse profissional não verificar o grau de entendimento do paciente sobre o diagnóstico recém recebido. ${ }^{21}$ Além disso, em mais da metade das consultas, os médicos não verificaram a compreensão do paciente sobre as indicações terapêuticas. ${ }^{22}$ 
Embora as relações entre equipes de saúde e usuários nem sempre permitam um diálogo maior ou mais franco, é válido lembrar que o papel dos portadores de DM, para que seu tratamento aconteça de forma adequada, não deve ser minimizado. Segundo o quinto princípio da Carta dos Direitos dos Usuários da Saúde do Ministério da Saúde (2007), "todo cidadão também tem responsabilidades para que seu tratamento aconteça da forma adequada" e deve "manifestar a compreensão sobre as informações elou orientações recebidas e, caso subsistam dúvidas, solicitar esclarecimento sobre elas". 23

O modelo biomédico estimula a separação entre o observador e o observado, ocasionando um distanciamento entre eles. Este modelo, predominante nos serviços de saúde, tem reduzido a visão da doença exclusivamente ao processo biológico e ocasiona dificuldades na comunicação entre o profissional de saúde e o usuário. ${ }^{10,11} \mathrm{O}$ profissional considera somente a doença em questão e não incorpora ao tratamento sentimentos e saberes do portador de DM. ${ }^{11,12}$ Fica evidente, a partir dessa constatação, que não ocorre um acordo entre eles, o que dificulta a adesão às orientações terapêuticas. O profissional tem que construir uma relação com o usuário para além da doença, agregando, ao fator biológico, características sociais e psicológicas. Por meio dessa prática comunicacional, passa a ser estabelecido um processo de trocas, com diálogo permanente, o qual será utilizado para persuadir o portador de DM a aderir ao tratamento. ${ }^{22}$

\section{Sobre orientações alimentares}

Em geral, as recomendações alimentares repassadas pelo serviço de saúde aos portadores de DM entrevistados visavam ao adequado controle dos níveis glicêmicos no sangue. Portanto, elas priorizavam a adoção de hábitos para uma alimentação saudável.

Ao serem questionados sobre as orientações recebidas, os usuários reportavam a existência de uma folha de recomendações alimentares, na qual estavam assinaladas as mais pertinentes a seu caso. Tal folha, em geral, estava guardada numa gaveta ou fixada na geladeira. Mesmo as pessoas que a colocaram na geladeira não davam maior atenção aos alimentos assinalados. Apesar das dificuldades para incorporarem às orientações na sua alimentação diária, o ato de não misturar os carboidratos complexos numa mesma refeição foi a orientação mais reportada pelos entrevistados e de maior aceitação dentre outras propostas pela equipe de saúde.

Quanto ao seguimento das recomendações alimentares, constatou-se que algumas pessoas tiveram sucesso após aderirem a elas, conseguindo incorporar no seu dia a dia as orientações. Todavia, eles relatam que, apesar de adquirirem novos hábitos alimentares, enfrentavam dificuldades para abandonar o costume de consumir carne gorda, banha e doces. A recomendação que se mostrou de mais difícil adesão é a de não consumir alimentos ricos em carboidratos simples; 
para adoçar as preparações, foi constantemente recomendada a todos a utilização de adoçantes dietéticos, segundo os entrevistados.

O problema, no entanto, não se encontra no fato de tornar doce uma preparação, e sim na aderência à interdição alimentar. O fato de se sentirem proibidos de comer alimentos aos quais estavam habituados é fator importante para o manejo correto da doença. Estudo feito por Maias Neto mostra que há obstáculos para a continuidade das dietas de portadores de DM. ${ }^{18}$ A preferência por determinados tipos de alimentos, o preparo das refeições e o preço dos alimentos foram entraves significativos para a adesão às orientações. ${ }^{16}$

A proibição, que atinge também as quantidades das porções de alimentos, dificulta o entendimento de que devem comer menos do que comiam anteriormente. Em geral, a restrição aos alimentos os levava a um ganho compensatório em situações de reunião social, como em festas. Estudo feito por Peres verificou que portadores de diabetes tiveram dificuldade no seguimento das orientações alimentares, visto que a dieta estava frequentemente associada a uma imagem negativa - isto é, "a perda do prazer de comer e beber, a restrição da autonomia e o cerceamento da liberdade para se alimentar como e quando desejar". ${ }^{24}$

\section{Fatores que dificultam a adesão às orientações alimentares}

Restrição alimentar. Os alimentos sugeridos nas orientações da equipe de saúde são conflitantes com vários aspectos da cultura local, principalmente no que tange a quantidade e qualidade a ser ingerida nas refeições. $\mathrm{O}$ valor cultural e o simbolismo criado sobre a presença e a grande quantidade diária de alguns alimentos nas refeições são determinantes da concepção de saúde da classe trabalhadora, geram força e dão sustento. ${ }^{24}$

A ideia de saúde, no universo estudado, liga-se à visão de corpulência e robustez, distanciandose da concepção de ingestão de alimentos leves e em pequenas porções durante o dia. Portanto, ingerir uma quantidade menor se associa a uma iminente fraqueza ou doença, dificultando a adesão às novas recomendações alimentares após o diagnóstico. Em estudo de caso realizado por Barsaglini, assim como no estudo de Maias Neto, a mesma concepção sobre a dieta foi relatada pelos entrevistados. ${ }^{18,25}$

Para os portadores de DM, a dieta preconizada a eles faz com que tenham de deixar de ser "bons de garfo". Como estas questões não são trabalhadas na UBS, podem ter afetado a adesão a elas. A concepção presente e destacada é de um empobrecimento da saúde, como ressalta Dona Hilda (64 anos): "Tenho um bom apetite, se fizer como a nutricionista manda, posso até pegar uma fraqueza...". Para evitar que, com a fraqueza (eminente) fique mais doente, ela não se considera adepta às recomendações e burla sua dieta, ingerindo mais do que o necessário para sua condição. 
Desejo e proibição. Como referido anteriormente, uma das orientações mais importantes e difíceis de obter adesão é a de não consumir doces e açúcares simples em geral. Os entrevistados relatam que o alimento proibido se torna mais cobiçado. Até mesmo aqueles que não sentiam muita vontade de consumir doces antes de se descobrirem portadores de DM, o desejavam após diagnóstico. Outros relataram que já consumiam doces habitualmente e, mesmo cientes do perigo a sua saúde atual, não resistiam à tentação.

Situação idêntica foi relatada nos estudos de Peres, realizado com mulheres portadoras de DM, usuárias de uma Unidade Básica de Saúde de Ribeirão Preto-SP. A autora cita Santana para explicar essa relação entre proibição e desejo:

[...] O sabor do proibido faz mais doce o mel. [...] Da proibição surge o desejo, e desse desejo surge à transgressão. Na verdade a proibição aguça o objeto do desejo, quanto mais proibido mais desejado se torna. [...] $O$ desejo alimentar está sempre presente na vida do indivíduo diabético. Esse mesmo desejo o faz sofrer, reprimir, salivar, esquecer, transgredir, mentir, negar, admitir, sentir prazer, controlar e sentir culpa.

A cidade de Pelotas é considerada a capital brasileira do doce; sua economia e mídia promovem constantemente o consumo de todos os tipos de doçuras, o que torna para os entrevistados o doce ainda mais presente e desejado. Ele é de fácil acesso, vendido em qualquer comércio. Além disso, é utilizado muitas vezes como fonte de renda de algumas famílias contatadas. É o caso do Sr. Carlos (56 anos), cujo relato abaixo reflete sua dificuldade.

Algumas modificações na minha alimentação foram possiveis de se fazer. Transformar a feijoada em feijão, diminuir a gordura, comer frutas e verduras. Mas o problema é o doce! Porque é o meu trabalho! Estou sempre em contato com ele, desde o preparo a venda e é difícil de resistir. Antes eu comia muito doce, hoje eu tento me controlar, mas de vez em quando eu como uma rapinha que fica na panela.

Dentro de uma concepção fetichista de consumo, se estabelecem relações propícias para que o doce atinja o status de felicidade e satisfação. Quando o objeto de desejo é a fonte de renda ou está associado a eventos sociais dos mais variados tipos, os portadores de DM se veem em meio a duas situações conflitantes: o consumo de alimentos "proibidos" e o convívio social.

Isolamento social. $\mathrm{O}$ ato de alimentar-se ultrapassa as barreiras do fisiológico; vai além de suprir as necessidades nutricionais; é uma necessidade também social. Os alimentos possuem significados sociais e culturais que traçam identidades e unem pessoas no dia a dia. ${ }^{26,27}$ Quase sempre, o alimento assume um papel social no que se refere às relações humanas. Práticas como as confraternizações, os encontros entre amigos, à ida ao cinema ou uma simples partida de futebol são momentos em que o consumo de alimentos agrega, traz prazer e produz sentidos para muitos grupos. 
Em relação aos encontros/festas sociais, os entrevistados relataram três diferentes reações: 1) alguns se privam, muitas vezes, do convívio de outras pessoas para não comerem doces e alimentos que não lhes são permitidos; 2) outros vão aos encontros e consomem os doces e bebidas, minimizando as preocupações com a doença; e 3) apenas dois entrevistados referiram adesão às recomendações tal como foram feitas, não comendo alimentos fora da dieta aconselhada.

Contudo, aqueles que vão com mais frequência aos encontros e consomem as doçuras, demonstram conhecimento sobre como seu corpo reage às proibições. Eles ingerem os alimentos em pequenas quantidades, ficando alertas para o aparecimento de algum sintoma (nenhum dos entrevistados soube quantificar exatamente essa porção).

Esses indivíduos, os quais transgridem as recomendações, são portadores de DM há mais de cinco anos e já estão familiarizados com os sintomas, a reação do corpo e o manejo da doença. Assim sendo, conseguem se aventurar pelo consumo de alimentos e/ou bebidas proibitivos. Foi interessante perceber que, mesmo desrespeitando a orientação, a maioria dos entrevistados se considera aderente às recomendações.

Aspectos financeiros. Dentre os aspectos dificultosos para o seguimento da dieta, estão as condições econômicas do usuário ou da sua família. Muitos usuários relatam a dificuldade financeira para variarem sua dieta e/ou usarem alimentos diet e light. Entre os entrevistados, também foi encontrada esta justificativa.

Os relatos abaixo demonstram, resumidamente, como eles defendem o fato de não incorporarem novos alimentos na sua dieta, reforçando a ideia de que alimentos adequados a sua doença não são para pobre. Diet e light são alimentos considerados de ricos, de pessoas que além do acesso a eles, podem ter um tratamento fora da rede pública e com maior atenção - quiçá, que entendam da origem da doença e conversem com seu médico.

O que acontece é que pobre não faz dieta... faz o que dá; come legumes e frutas quando dá pra comprar... (Lucas, 55 anos).

É difícil ter uma alimentação balanceada. O pobre faz o que consegue comprar, os alimentos diet são muitos caros, as frutas e verduras também... (Juliana, 51 anos).

Eu vou levando conforme a minh a condição. Eu gostaria de fazer uma super alimentação, com alimentos diet e light, mas eu não posso é muito caro, então eu vou levando como dá... (Luciano, 50 anos).

Mesmo levando-se em consideração a influência do poder aquisitivo na compra de frutas, verduras e alimentos dietéticos, justificativa bastante utilizada pelos entrevistados para modificar as recomendações alimentares, deve-se questionar o conceito de que uma alimentação saudável é privilégio somente de pessoas com elevada condição financeira. Para isso, é preciso discutir a ideia 
de que os altos preços de alimentos orgânicos são fatores preponderantes para sua não utilização nas refeições diárias de famílias de baixa renda.

\section{Conclusões}

A complexidade do manejo da DM é um grande desafio para o sistema de saúde altamente sobrecarregado. Todavia, toda forma de intervenção e de educação em saúde para DM deve considerar que o autocuidado está relacionado a questões nem sempre evidentes no momento da consulta ou do atendimento em grupo do Hiperdia. Avaliar novas formas de controle e de comunicação deve ser um compromisso constante na atenção básica que busca ofertar uma saúde pública universal e integral.

O presente estudo desperta a importância da discussão sobre o modelo de atenção hegemônico vigente em nosso país. É preciso que profissionais de saúde, prestadores de serviço, usuários e gestores se voltem para o debate, procurando gerar soluções para modificar o atual modelo. Está comprovado que a racionalidade do atendimento utilizada como guia para a atenção em saúde não responde às necessidades dos usuários e dificulta a ação dos profissionais de saúde. ${ }^{13}$

Dentro desse contexto, surge o Programa Saúde da Família (PSF) na década de 1990, o qual postula a tentativa de reorientar o modelo assistencial a partir da atenção básica. Outros programas - a exemplo do Plano de Reorganização da Atenção à Hipertensão Arterial e ao Diabetes Mellitus e o Sistema de Cadastramento e Acompanhamento de Hipentensos e Diabéticos - surgem para reforçar essa mudança e melhorar a qualidade da atenção à saúde. ${ }^{5}$ Através do Hiperdia, é possível obter um cadastro de hipertensos e diabéticos, a fim de alcançar a identificação precoce dos casos e estabelecer um vínculo com as UBS e equipes de saúde, ambos, elementos indispensáveis para o controle de agravos relacionados a essas doenças e à adesão ao tratamento.

Contudo, apesar dos esforços empreendidos pelos governantes através de políticas de capacitação dos gestores e profissionais para atuarem na área da saúde, ainda não é possível perceber mudanças efetivas no modelo de atenção. O modelo tradicional de educação em saúde considera os usuários como indivíduos carentes de informação nesta área, fazendo com que a comunicação entre o profissional e o usuários seja vertical, onde o primeiro determina o tratamento a ser seguido e cabe ao segundo apenas executá-lo. Além disso, ele não considera os fatores psicossociais e culturais determinantes do comportamento em saúde. ${ }^{25}$ 
Como demonstrado neste estudo, a tática utilizada para estimular a adesão às recomendações não consegue alcançar êxito, visto que o modelo de educação tradicional, o qual está sendo utilizado nos serviços de saúde, não atinge a vida cotidiana dos usuários - ou seja, possui apenas caráter informativo e não é capaz de transformar saberes existentes em práticas de saúde.

Para confrontar esse modo de educação em saúde, surge o modelo dialógico que tem o diálogo como seu instrumento essencial, no qual o usuário é possuidor de um saber considerado na interação médico-usuário. Através desse modelo, é possível aproximar o usuário do sistema de saúde e dos profissionais, tornando efetivo o tratamento e a adesão a ele. ${ }^{25}$ Todavia, diante de sua importância, este modelo tem sido pouco aplicado na atenção básica, ainda que tenha gerado debate no meio, especialmente entre os que estudam educação em saúde. Tal modelo exige transformações que levariam a novas posturas profissionais, o que provavelmente poderia gerar resistências e discordâncias de várias formas.

Desconsiderar ou não valorizar que há um sistema de valores, símbolos e significados "leigos" dados à comida e ao se alimentar, distintos dos não médicos, coloca a dimensão do comer como uma prática de sobrevivência apenas. Uma melhor compreensão dos hábitos e práticas alimentares por parte dos profissionais de saúde poderá levar a uma maior eficácia das orientações e interação com os usuários portadores de DM, que não raramente são aconselhados a se submeter a um controle alimentar muito distinto do seu habitual.

Cabe ressaltar ainda, mesmo sendo a UBS estudada uma unidade de ensino e da qual fazem parte alunos com formação parcial em nutrição, os estímulos efetivos para a inserção de hábitos saudáveis e para a adesão às orientações alimentares são pouco trabalhados, conforme os depoimentos dos portadores de DM. Diante disso, ações que visem à educação em saúde poderiam ser também priorizadas, considerando os aspectos socioculturais que influenciam na adesão e no manejo do tratamento para a DM.

Deste estudo, certamente restaram lacunas a serem preenchidas, assim como alguns aspectos que não foram abordados e que podem influenciar na não adesão às orientações alimentares. Entre esses, se insere a visão dos portadores de DM sobre a questão do açúcar no sangue, os sentimentos decorrentes da doença e o entendimento de como os mecanismos biológicos agem e podem ajudar no tratamento da doença. É preciso aprofundar a visão sobre a compreensão da doença e do tratamento, as causas do DM e, ainda, as questões que levam à não adesão do ponto de vista dos portadores da doença. 


\section{Referências}

1. Peres DS, Santos MA, Zanetti ML, Ferronato AA. Dificuldades dos pacientes diabéticos para o controle da doença: Sentimentos e comportamentos. Rev. Latino-Am. Enfermagem 2007; 15 (6):1-8.

2. Reis OM, Bachion MM, Nakatani AYK. Preparo de médicos para o atendimento aos diabéticos no Programa Saúde da Família e suas percepções sobre as dificuldades de adesão ao tratamento. Acta Sci. Health Sci. 2005; 27(2):119-129.

3. Sartorelli DS, Franco LJ. Tendências do diabetes mellitus no Brasil: o papel da transição nutricional. Cad. Saúde Pública 2003; 19(Suppl.1):29-36.

4. Dias da Costa JS, Olinto MTA, Assunção MCF, Gigante DP, Macedo S, Menezes AMB. Prevalência de Diabetes Mellitus em Pelotas, RS: um estudo de base populacional. Rev Saúde Pública 2006; 40(3):542-5.

5. Brasil. Ministério da Saúde. Secretaria de Políticas Públicas. Plano de Reorganização da Atenção à Hipertensão Arterial e ao Diabetes Mellitus. Rev. Saúde Pública 2001; 35(6):585-588.

6. Brasil. Ministério da Saúde. Diabetes Mellitus. Cadernos de Atenção Básica, n. 16. Brasília: MS; 2006.

7. Apóstolo JLA, Viveiros CSC, Nunes HIR, Domingues HRF. Incerteza na doença e motivação para o tratamento em diabéticos tipo 2. Rev. Latino-Am. Enfermagem 2007; 15(4):1-9.

8. Assis MAA, Nahas MV. Aspectos motivacionais em programas de mudança de comportamento alimentar. Rev. Nutr. Campinas, 1999; 12(1):33-41.

9. Portero KCC, Cattalini M. Mudança no estilo de vida para prevenção e tratamento do Diabetes Mellitus tipo 2. Rev. Saúde de Piracicaba 2005; 7(16):63-69.

10. Brasil. Conselho Nacional de Saúde. Resolução 196/96. Diretrizes e normas regulamentadoras em pesquisa envolvendo seres humanos. Brasília: Ministério da Saúde; 1996.

11. Wise, B. In their own words: the lived experience of pediatric liver transplantation. Thousand Oaks. Qualitative Health Research 2002; 12(1):74-90.

12. Traverso-Yépez M, Morais NA. Reivindicando a subjetividade dos usuários da Rede Básica de Saúde: para uma humanização do atendimento. Cad. Saúde Pública 2004; 20(1):80-88.

13. Oliveira FA. Antropologia nos serviços de saúde: integralidade, cultura e comunicação. Interface Comunic, Saúde, Educ. 2002; 6(10):63-74.

14. Cecílio LCO. Inventando a mudança na saúde. São Paulo: Hucitec; 1997.

15. Gonçalves H, Dias da Costa JS, Menezes AMB, Knauth D, Leal OF. Adesão à terapêutica a tuberculose em Pelotas, Rio Grande do Sul: na perspectiva do paciente. Cad. Saúde Pública 1999; 15(4):777-787.

16. Barros JAC. Pensando o processo saúde e doença: a que responde o modelo biomédico. Saúde e Sociedade 2002; 11(1):67-84.

17. Welfer M, Leite MT. Ser portador de diabetes tipo 2: cuidando-se para continuar vivendo. Scientia Medica 2005; 15(3):148-155. 
18. Maias Neto RC. Vivendo com o diabetes mellitus: a experiência dos sujeitos atendidos em uma unidade publica de saúde no Rio de Janeiro [dissertação]. Rio de Janeiro: Escola Nacional de Saúde Publica; Fundação Oswaldo Cruz; 2003.

19. Zahar J. Da Vida nervosa (nas classes trabalhadoras urbanas). Rio de Janeiro: CNPq; 1986.

20. Duarte LFD, Leal OF. Doença, sofrimento, perturbação: perspectivas etnográficas. Rio de Janeiro; Editora Fiocruz; 1998.

21. Caprara A, Rodrigues J. A relação assimétrica médico-paciente: repensando o vínculo terapêutico. Cien. Saúde Colet. 2004; 9(1):139-146.

22. Caprara A, Silva Franco AL. A Relação paciente-médico: para uma humanização da prática médica Cad. Saúde Pública 1999; 15(3):647-654.

23. Brasil. Ministério da Saúde. Carta dos direitos dos usuários da saúde. Brasília: Ministério da Saúde; 2006. 8 p.

24. Peres DS, Franco LJ, Antônio dos Santos M. Comportamento alimentar em mulheres portadoras de diabetes tipo 2. Rev. Saúde Pública 2006; 40(2):310-7.

25. Barsaglini RA. Com açúcar no sangue até o fim: um estudo de caso sobre o viver com Diabetes. In: Canesqui AM. Olhares socioantropológicos sobre os adoecidos crônicos. Hucitec; 2007.

26. Jaime PC, Machado FS, Westphal MF; Monteiro, CA. Educação nutricional e consumo de frutas e hortaliças: ensaio comunitário controlado. Rev. Saúde Pública 2007; 41(1):154-157.

27. Alves VS. Um modelo de educação em saúde para o Programa Saúde da Família: pela integralidade da atenção é reorientação do modelo assistencial. Interface - Comunic, Saúde, Educ 2005; 9 (16):39-52.

Recebido: 06/1/2014

Revisado: 19/3/2014

Aprovado: 25/4/2014 
